PROCEEDINGS OF THE

AMERICAN MATHEMATICAL SOCIETY

Volume 138, Number 2, February 2010, Pages 753-758

S 0002-9939(09)10124-7

Article electronically published on September 30, 2009

\title{
A NOTE ON THE ABELIANIZATIONS OF FINITE-INDEX SUBGROUPS OF THE MAPPING CLASS GROUP
}

\author{
ANDREW PUTMAN \\ (Communicated by Daniel Ruberman)
}

\begin{abstract}
For some $g \geq 3$, let $\Gamma$ be a finite index subgroup of the mapping class group of a genus $g$ surface (possibly with boundary components and punctures). An old conjecture of Ivanov says that the abelianization of $\Gamma$ should be finite. In the paper we prove two theorems supporting this conjecture. For the first, let $T_{x}$ denote the Dehn twist about a simple closed curve $x$. For some $n \geq 1$, we have $T_{x}^{n} \in \Gamma$. We prove that $T_{x}^{n}$ is torsion in the abelianization of $\Gamma$. Our second result shows that the abelianization of $\Gamma$ is finite if $\Gamma$ contains a "large chunk" (in a certain technical sense) of the Johnson kernel, that is, the subgroup of the mapping class group generated by twists about separating curves.
\end{abstract}

\section{INTRODUCTION}

Let $\Sigma_{g, b}^{p}$ be an oriented genus $g$ surface with $b$ boundary components and $p$ punctures and let $\operatorname{Mod}\left(\Sigma_{g, b}^{p}\right)$ be its mapping class group, that is, the group of isotopy classes of orientation-preserving diffeomorphisms of $\Sigma_{g, b}^{p}$ that fix the boundary components and punctures pointwise (we will omit $b$ or $p$ when they are zero). A long-standing conjecture of Ivanov (see [6] for a recent discussion) says that for $g \geq 3$, the group $\operatorname{Mod}\left(\Sigma_{g, b}^{p}\right)$ does not virtually surject onto $\mathbb{Z}$. In other words, if $\Gamma$ is a finite-index subgroup of $\operatorname{Mod}\left(\Sigma_{g, b}^{p}\right)$, then $\mathrm{H}_{1}(\Gamma ; \mathbb{R})=0$.

The goal of this paper is to offer some evidence for this conjecture. If $G$ is a group and $g \in G$, then we will denote by $[g]_{G}$ the corresponding element of $\mathrm{H}_{1}(G ; \mathbb{R})$. Also, for a simple closed curve $\gamma$ on $\Sigma_{g, b}^{p}$, we will denote by $T_{\gamma}$ the corresponding right Dehn twist. Observe that if $\Gamma$ is any finite-index subgroup of $\operatorname{Mod}_{g, b}^{p}$, then $T_{\gamma}^{n} \in \operatorname{Mod}_{g, b}^{p}$ for some $n \geq 1$. Our first result is the following.

Theorem A (Powers of twists vanish). For some $g \geq 3$, let $\Gamma<\operatorname{Mod}\left(\Sigma_{g, b}^{p}\right)$ satisfy $\left[\operatorname{Mod}\left(\Sigma_{g, b}^{p}\right): \Gamma\right]<\infty$ and let $\gamma$ be a simple closed curve on $\Sigma_{g, b}^{p}$. Pick $n \geq 1$ such that $T_{\gamma}^{n} \in \Gamma$. Then $\left[T_{\gamma}^{n}\right]_{\Gamma}=0$.

Remark. After this paper was written, Bridson informed us that in unpublished work he had proven a result about mapping class group actions on CAT(0) spaces that implies Theorem $\mathrm{A}$ Bridson's work will appear in [3].

Received by the editors February 3, 2009, and, in revised form, May 19, 2009.

2000 Mathematics Subject Classification. Primary 57-XX; Secondary 20-XX.

(C)2009 American Mathematical Society Reverts to public domain 28 years from publication 
We use this to verify Ivanov's conjecture for a class of examples. For a long time, the only positive evidence for Ivanov's conjecture was a result of Hain [5] that says that it holds for all finite-index subgroups containing the Torelli group $\mathcal{I}_{g, b}^{p}$, that is, the kernel of the action of $\operatorname{Mod}\left(\Sigma_{g, b}^{p}\right)$ on $\mathrm{H}_{1}\left(\Sigma_{g} ; \mathbb{Z}\right)$ induced by filling in all the punctures and boundary components. The group $\mathcal{I}_{g, b}^{p}$ contains the Johnson kernel $\mathcal{K}_{g, b}^{p}$, which is the subgroup generated by Dehn twists about separating curves. A result of Johnson [7] says that $\mathcal{K}_{g, b}^{p}$ is an infinite-index subgroup of $\mathcal{I}_{g, b}^{p}$.

For a subgroup $\Gamma$ of $\operatorname{Mod}\left(\Sigma_{g, b}^{p}\right)$, denote by $K(\Gamma)$ the subgroup of $\Gamma \cap \mathcal{K}_{g, b}^{p}$ generated by the set

$$
\left\{T_{\gamma}^{n} \mid \gamma \text { a separating curve, } n \in \mathbb{Z} \text {, and } T_{\gamma}^{n} \in \Gamma\right\} .
$$

If $\mathcal{K}_{g, b}^{p}<\Gamma$, then $K(\Gamma)=\Gamma \cap \mathcal{K}_{g, b}^{p}$, but the converse does not hold. Our second result is the following.

Theorem B (Subgroups containing large pieces of Johnson kernel). For some $g \geq 3$, let $\Gamma<\operatorname{Mod}\left(\Sigma_{g, b}^{p}\right)$ satisfy $\left[\operatorname{Mod}\left(\Sigma_{g, b}^{p}\right): \Gamma\right]<\infty$. Assume that $\left[\Gamma \cap \mathcal{K}_{g, b}^{n}\right.$ : $K(\Gamma)]<\infty$. Then $\mathrm{H}_{1}(\Gamma ; \mathbb{R})=0$.

As a corollary, we obtain the following result, which was recently proven by Boggi [2] via a difficult algebro-geometric argument under the assumption $b=p=0$.

Corollary C (Subgroups containing Johnson kernel). For some $g \geq 3$, let $\Gamma<$ $\operatorname{Mod}\left(\Sigma_{g, b}^{p}\right)$ satisfy $\left[\operatorname{Mod}\left(\Sigma_{g, b}^{p}\right): \Gamma\right]<\infty$. Assume that $\mathcal{K}_{g, b}^{n}<\Gamma$. Then $\mathrm{H}_{1}(\Gamma ; \mathbb{R})=0$.

Remark. McCarthy [1] proved that Ivanov's conjecture fails in the case $g=2$.

\section{NOTATION AND BASIC FACTS ABOUT GROUP HOMOLOGY}

If $M$ is a $G$-module, then $M_{G}$ will denote the coinvariants of the action, that is, the quotient of $M$ by the submodule generated by the set $\{x-g(x) \mid x \in M, g \in G\}$. This appears in the 5-term exact sequence [4, Corollary VII.6.4], which asserts the following. If

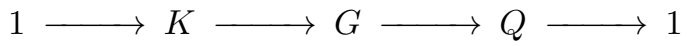

is a short exact sequence of groups, then for any ring $R$, there is an exact sequence

$$
\mathrm{H}_{2}(G ; R) \longrightarrow \mathrm{H}_{2}(Q ; R) \longrightarrow\left(\mathrm{H}_{1}(K ; R)\right)_{Q} \longrightarrow \mathrm{H}_{1}(G ; R) \longrightarrow \mathrm{H}_{1}(Q ; R) \longrightarrow 0 \text {. }
$$

If $G_{2}<G_{1}$ are groups satisfying $\left[G_{1}: G_{2}\right]<\infty$ and $R$ is a ring, then for all $k$ there exists a transfer map of the form $t: \mathrm{H}_{k}\left(G_{1} ; R\right) \rightarrow \mathrm{H}_{k}\left(G_{2} ; R\right.$ ) (see, e.g., [4, Chapter III.9]). The key property of $t$ (see [4, Proposition III.9.5]) is that if $i: \mathrm{H}_{k}\left(G_{2} ; R\right) \rightarrow \mathrm{H}_{k}\left(G_{1} ; R\right)$ is the map induced by the inclusion, then $i \circ t: \mathrm{H}_{k}\left(G_{1} ; R\right) \rightarrow \mathrm{H}_{k}\left(G_{1} ; R\right)$ is multiplication by $\left[G_{1}: G_{2}\right]$. In particular, if $R=\mathbb{R}$, then we obtain a right inverse $\frac{1}{\left[G_{1}: G_{2}\right]} t$ to $i$. This yields the following standard lemma.

Lemma 2.1. Let $G_{2}<G_{1}$ be groups satisfying $\left[G_{1}: G_{2}\right]<\infty$. For all $k$, the map $\mathrm{H}_{k}\left(G_{2} ; \mathbb{R}\right) \rightarrow \mathrm{H}_{k}\left(G_{1} ; \mathbb{R}\right)$ is surjective. 


\section{Proof of Theorem $\mathrm{A}$}

Let $n \geq 1$ be the smallest integer such that $T_{\gamma}^{n} \in \Gamma$.

We first claim that there exists a subsurface $S \hookrightarrow \Sigma_{g, b}^{p}$ whose genus is at least 2 with the following property. Let $i: \operatorname{Mod}(S) \rightarrow \operatorname{Mod}\left(\Sigma_{g, b}^{p}\right)$ be the induced map ("extend by the identity"). Then there exists some boundary component $\beta$ of $S$ such that $i\left(T_{\beta}\right)=T_{\gamma}$. There are two cases. If $\gamma$ is nonseparating, then let $S$ be the complement of a regular neighborhood of $\gamma$. Observe that $S \cong \Sigma_{g-1, b+2}^{p}$, so the genus of $S$ is at least 2. If instead $\gamma$ is separating, then let $S$ be the component of $\Sigma_{g, b}^{p}$ cut along $\gamma$ whose genus is maximal. Since $g \geq 3$, this subsurface must have genus at least 2 . The claim follows.

Define $\Gamma^{\prime}=i^{-1}(\Gamma)$. We have $T_{\beta}^{n} \in \Gamma^{\prime}$, and it is enough to show that $\left[T_{\beta}^{n}\right]_{\Gamma^{\prime}}=0$. Let $\bar{S}$ be the result of gluing a punctured disc to $\beta$ and let $\bar{\Gamma}^{\prime}$ be the image of $\Gamma^{\prime}$ in $\operatorname{Mod}(\bar{S})$. There is a diagram of central extensions

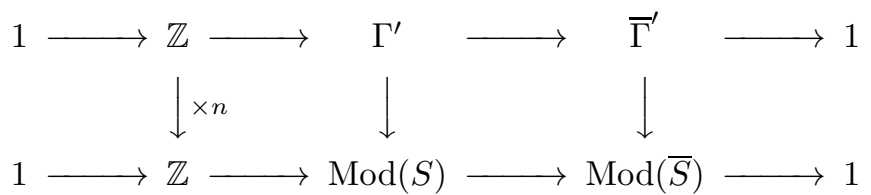

with $\mathbb{Z}<\operatorname{Mod}(S)$ and $\mathbb{Z}<\Gamma^{\prime}$ generated by $T_{\beta}$ and $T_{\beta}^{n}$, respectively. The last 4 terms of the corresponding diagram of 5 -term exact sequences are

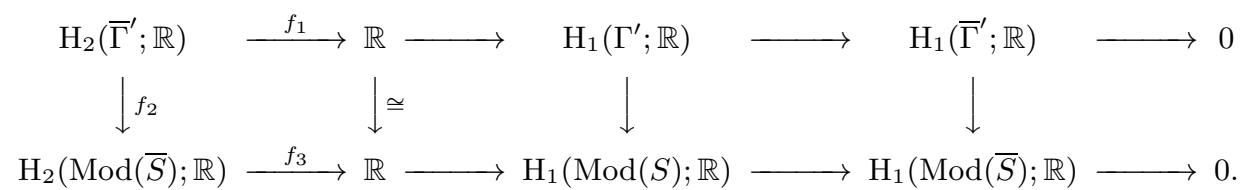

We remark that there are no nontrivial coinvariants in these sequences since our extensions are central. We must show that $f_{1}$ is a surjection. Since $S$ has genus at least 2 , we have $\mathrm{H}_{1}(\operatorname{Mod}(S) ; \mathbb{R})=0$ (see, e.g., [10]), so $f_{3}$ is a surjection. Since $\left[\operatorname{Mod}(\bar{S}): \bar{\Gamma}^{\prime}\right]<\infty$, Lemma 2.1 implies that $f_{2}$ is a surjection, so $f_{1}$ is a surjection, as desired.

\section{Proof of Theorem B}

4.1. Two facts about $\operatorname{Sp}_{2 g}(\mathbb{Z})$. We will need two standard facts about finite-index subgroups $\Gamma$ of $\operatorname{Sp}_{2 g}(\mathbb{Z})$, both of which follow from the fact that $\Gamma$ is a lattice in $\mathrm{Sp}_{2 g}(\mathbb{R})$.

For the first, since $\mathrm{Sp}_{2 g}(\mathbb{R})$ is a connected simple Lie group with finite center and real rank $g$, the group $\Gamma$ has Kazhdan's property (T) when $g \geq 2$ (see, e.g., 13, Theorem 7.1.4]). One standard property of groups with property $(\mathrm{T})$ is that they have no nontrivial homomorphisms to $\mathbb{R}$ (see, e.g., [13, Theorem 7.1.7]). Combining these facts, we obtain the following theorem.

Theorem 4.1. For some $g \geq 2$, let $\Gamma<\operatorname{Sp}_{2 g}(\mathbb{Z})$ satisfy $\left[\operatorname{Sp}_{2 g}(\mathbb{Z}): \Gamma\right]<\infty$. Then $\mathrm{H}_{1}(\Gamma ; \mathbb{R})=0$.

For the second, since $\mathrm{Sp}_{2 g}(\mathbb{R})$ is a connected noncompact simple real algebraic group, we can apply the Borel density theorem (see, e.g., [13, Theorem 3.2.5]) to deduce that $\Gamma$ is Zariski dense in $\operatorname{Sp}_{2 g}(\mathbb{R})$. This implies that any finite-dimensional 
nontrivial irreducible $\mathrm{Sp}_{2 g}(\mathbb{R})$-representation $V$ must also be an irreducible $\Gamma$ representation; indeed, if $V^{\prime}$ was a nontrivial proper $\Gamma$-submodule of $V$, then the subgroup of $\mathrm{Sp}_{2 g}(\mathbb{R})$ preserving $V^{\prime}$ would be a proper subvariety of $\mathrm{Sp}_{2 g}(\mathbb{R})$ containing $\Gamma$. Recall that the ring of coinvariants $V_{\Gamma}$ of $V$ under $\Gamma$ is the quotient $V / K$, where $K=\langle x-g(x) \mid x \in V, g \in \Gamma\rangle$. Since $K \neq 0$, we can apply Schur's lemma to deduce that $K=V$, i.e. that $V_{\Gamma}=0$. We record this fact as the following theorem.

Theorem 4.2. For some $g \geq 1$, let $\Gamma<\operatorname{Sp}_{2 g}(\mathbb{Z})$ satisfy $\left[\operatorname{Sp}_{2 g}(\mathbb{Z}): \Gamma\right]<\infty$ and let $V$ be a nontrivial irreducible $\mathrm{Sp}_{2 g}(\mathbb{R})$-representation. Then $V_{\Gamma}=0$.

4.2. Two preliminary lemmas. We will need two lemmas. The first is the following, which slightly generalizes a theorem of Johnson 8 .

Lemma 4.3. For $g \geq 3$, we have $\mathcal{I}_{g, b}^{p} / \mathcal{K}_{g, b}^{p} \cong\left(\wedge^{3} H\right) / H \oplus H^{b+p}$, where $H=$ $\mathrm{H}_{1}\left(\Sigma_{g} ; \mathbb{Z}\right)$.

Proof. Since $\mathcal{K}_{g, b}^{p}$ contains all twists about boundary curves, we can assume that $b=0$.

Building on work of Johnson [9], Hain [5] proved that

$$
\mathrm{H}_{1}\left(\mathcal{I}_{g}^{p} ; \mathbb{R}\right) \cong\left(\wedge^{3} H_{\mathbb{R}}\right) / H_{\mathbb{R}} \oplus H_{\mathbb{R}}^{p},
$$

where $H_{\mathbb{R}}=\mathrm{H}_{1}\left(\Sigma_{g} ; \mathbb{R}\right)$. Also, Johnson [9, Lemma 2] proved that for $x \in \mathcal{K}_{g}^{p}$, we have $[x]_{\mathcal{I}_{q}^{p}}=0$. (Johnson only considered the case where $p=0$, but his argument works in general.) It follows that

$$
\mathrm{H}_{1}\left(\mathcal{I}_{g}^{p} / \mathcal{K}_{g}^{p} ; \mathbb{R}\right) \cong\left(\wedge^{3} H_{\mathbb{R}}\right) / H_{\mathbb{R}} \oplus H_{\mathbb{R}}^{p} .
$$

We will prove the lemma by induction on $p$. The base case $p=0$ is a theorem of Johnson [8. Assume now that $p>0$ and that the lemma is true for all smaller $p$. Fixing a puncture $*$ of $\Sigma_{g}^{p}$, work of Birman [1] and Johnson [9] gives an exact sequence

$$
1 \longrightarrow \pi_{1}\left(\Sigma_{g}^{p-1}, *\right) \longrightarrow \mathcal{I}_{g}^{p} \longrightarrow \mathcal{I}_{g}^{p-1} \longrightarrow 1,
$$

where the map $\mathcal{I}_{g}^{p} \rightarrow \mathcal{I}_{g}^{p-1}$ comes from "forgetting the puncture $*$ ". Quotienting out by $\mathcal{K}_{g}^{p}$, we obtain an exact sequence

$$
1 \longrightarrow \pi_{1}\left(\Sigma_{g}^{p-1}, *\right) /\left(\pi_{1}\left(\Sigma_{g}^{p-1}, *\right) \cap \mathcal{K}_{g}^{p}\right) \longrightarrow \mathcal{I}_{g}^{p} / \mathcal{K}_{g}^{p} \longrightarrow \mathcal{I}_{g}^{p-1} / \mathcal{K}_{g}^{p-1} \longrightarrow 1
$$

By induction, we have

$$
\mathcal{I}_{g}^{p-1} / \mathcal{K}_{g}^{p-1} \cong\left(\wedge^{3} H\right) / H \oplus H^{p-1} .
$$

Set $A=\pi_{1}\left(\Sigma_{g}^{p-1}, *\right) /\left(\pi_{1}\left(\Sigma_{g}^{p-1}, *\right) \cap \mathcal{K}_{g}^{p}\right)$. We will prove that $A$ is a quotient of $H$. We will then be able to conclude that $\mathcal{I}_{g}^{p-1} / \mathcal{K}_{g}^{p-1}$ acts trivially on $A$, so $\mathcal{I}_{g}^{p} / \mathcal{K}_{g}^{p}$ is the abelian group

$$
\left(\wedge^{3} H\right) / H \oplus H^{p-1} \oplus A .
$$

Using (1), a simple dimension count will then imply that $A$ cannot be a proper quotient of $H$, and the lemma will follow.

The element of $\mathcal{I}_{g}^{p}$ corresponding to $\delta \in \pi_{1}\left(\Sigma_{g}^{p-1}, *\right)$ "drags" * around $\delta$. As shown in Figure 1, a-b, a simple closed curve $\gamma \in \pi_{1}\left(\Sigma_{g}^{p-1}, *\right)$ corresponds to $T_{\gamma_{1}} T_{\gamma_{2}}^{-1} \in \mathcal{I}_{g}^{p}$, where $\gamma_{1}$ and $\gamma_{2}$ are the boundary components of a regular neighborhood of $\gamma$. In particular, if $\gamma$ is a simple closed separating curve, then as shown in Figure 1 $\mathrm{c}-\mathrm{d}$, the corresponding element of $\mathcal{I}_{g}^{p}$ is a product of separating twists. Since $\left[\pi_{1}\left(\Sigma_{g}^{p-1}, *\right), \pi_{1}\left(\Sigma_{g}^{p-1}, *\right)\right]$ is generated by simple closed separating curves (see, 

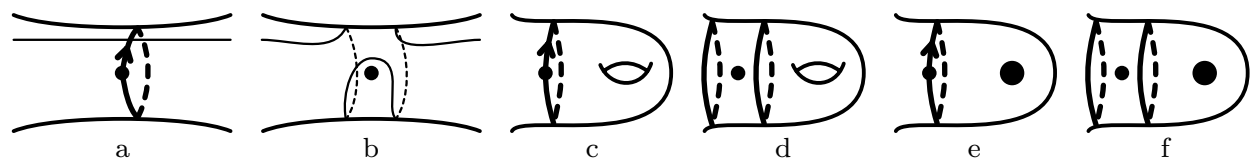

Figure 1. a-f. Curves needed for proof of Lemma 4.3

e.g., [12, Lemma A.1]), we deduce that $\left[\pi_{1}\left(\Sigma_{g}^{p-1}, *\right), \pi_{1}\left(\Sigma_{g}^{p-1}, *\right)\right] \subset \pi_{1}\left(\Sigma_{g}^{p-1}, *\right) \cap \mathcal{K}_{g}^{p}$. Thus $A=\pi_{1}\left(\Sigma_{g}^{p-1}, *\right) /\left(\pi_{1}\left(\Sigma_{g}^{p-1}, *\right) \cap \mathcal{K}_{g}^{p}\right)$ is a quotient of $\mathrm{H}_{1}\left(\Sigma_{g}^{p-1} ; \mathbb{Z}\right)$. Finally, as shown in Figure 1. $\mathrm{e}-\mathrm{f}$, all simple closed curves that are homotopic into punctures are also contained in $\pi_{1}\left(\Sigma_{g}^{p-1}, *\right) \cap \mathcal{K}_{g}^{p}$, so we conclude that $A$ is a quotient of $H=\mathrm{H}_{1}\left(\Sigma_{g} ; \mathbb{Z}\right)$, as desired.

For the second lemma, define $Q_{g, b}^{p}=\operatorname{Mod}_{g, b}^{p} / \mathcal{K}_{g, b}^{p}$.

Lemma 4.4. For some $g \geq 3$, let $Q^{\prime}<Q_{g, b}^{p}$ satisfy $\left[Q_{b, b}^{p}: Q^{\prime}\right]<\infty$. Then $\mathrm{H}_{1}\left(Q^{\prime} ; \mathbb{R}\right)=0$.

Proof. Restricting the short exact sequence

$$
1 \longrightarrow \mathcal{I}_{g, b}^{p} / \mathcal{K}_{g, b}^{p} \longrightarrow Q_{b, b}^{p} \longrightarrow \operatorname{Sp}_{2 g}(\mathbb{Z}) \longrightarrow 1
$$

to $Q^{\prime}$, we obtain a short exact sequence

$$
1 \longrightarrow B \longrightarrow Q^{\prime} \longrightarrow \bar{Q}^{\prime} \longrightarrow 1,
$$

where $B$ and $\bar{Q}^{\prime}$ are finite-index subgroups of $\mathcal{I}_{g, b}^{p} / \mathcal{K}_{g, b}^{p}$ and $\operatorname{Sp}_{2 g}(\mathbb{Z})$, respectively. The last 3 terms of the associated 5 -term exact sequence are

$$
\left(\mathrm{H}_{1}(B ; \mathbb{R})\right)_{\bar{Q}^{\prime}} \longrightarrow \mathrm{H}_{1}\left(Q^{\prime} ; \mathbb{R}\right) \longrightarrow \mathrm{H}_{1}\left(\bar{Q}^{\prime} ; \mathbb{R}\right) \longrightarrow 0
$$

By Theorem 4.1, we have $\mathrm{H}_{1}\left(\bar{Q}^{\prime} ; \mathbb{R}\right)=0$. Letting $H=\mathrm{H}_{1}\left(\Sigma_{g} ; \mathbb{Z}\right)$, Lemma 4.3 says that

$$
\mathcal{I}_{g, b}^{p} / \mathcal{K}_{g, b}^{p} \cong\left(\wedge^{3} H\right) / H \oplus H^{b+p} .
$$

Since $B$ is a finite-index subgroup of $\mathcal{I}_{g, b}^{p} / \mathcal{K}_{g, b}^{p}$, we get that $B$ is itself abelian and

$$
\mathrm{H}_{1}(B ; \mathbb{R}) \cong B \otimes \mathbb{R} \cong\left(\mathcal{I}_{g, b}^{p} / \mathcal{K}_{g, b}^{p}\right) \otimes \mathbb{R} \cong\left(\wedge^{3} H_{\mathbb{R}}\right) / H_{\mathbb{R}} \oplus H_{\mathbb{R}}^{b+p},
$$

where $H_{\mathbb{R}}=\mathrm{H}_{1}\left(\Sigma_{g} ; \mathbb{R}\right)$. Both $\left(\wedge^{3} H_{\mathbb{R}}\right) / H_{\mathbb{R}}$ and $H_{\mathbb{R}}$ are nontrivial finite-dimensional irreducible representations of $\mathrm{Sp}_{2 g}(\mathbb{R})$, so Theorem 4.2 implies that $\left(\mathrm{H}_{1}(B ; \mathbb{R})\right)_{\bar{Q}^{\prime}}=$ 0 , and we are done.

4.3. The proof of Theorem B. The last 3 terms of the 5 -term exact sequence associated to the short exact sequence

$$
1 \longrightarrow \Gamma \cap \mathcal{K}_{g, b}^{p} \longrightarrow \Gamma \longrightarrow \Gamma /\left(\Gamma \cap \mathcal{K}_{g, b}^{p}\right) \longrightarrow 1
$$

are

$$
\left(\mathrm{H}_{1}\left(\Gamma \cap \mathcal{K}_{g, b}^{p} ; \mathbb{R}\right)\right)_{\Gamma /\left(\Gamma \cap \mathcal{K}_{g, b}^{p}\right)} \stackrel{i}{\longrightarrow} \mathrm{H}_{1}(\Gamma ; \mathbb{R}) \longrightarrow \mathrm{H}_{1}\left(\Gamma /\left(\Gamma \cap \mathcal{K}_{g, b}^{p}\right) ; \mathbb{R}\right) \longrightarrow 0
$$

By assumption, $\left[\Gamma \cap \mathcal{K}_{g, b}^{p}: K(\Gamma)\right]<\infty$, so Lemma 2.1 implies that the map $\mathrm{H}_{1}(K(\Gamma) ; \mathbb{R}) \rightarrow \mathrm{H}_{1}\left(\Gamma \cap \mathcal{K}_{g, b}^{p} ; \mathbb{R}\right)$ is surjective. Since $K(\Gamma)$ is generated by powers of twists, Theorem $\mathrm{A}$ allows us to deduce that $i=0$. Also, $\Gamma /\left(\Gamma \cap \mathcal{K}_{g, b}^{p}\right)$ is a finite-index subgroup of $Q_{g, b}^{p}$, so Lemma 4.4 implies that $\mathrm{H}_{1}\left(\Gamma /\left(\Gamma \cap \mathcal{K}_{g, b}^{p}\right) ; \mathbb{R}\right)=0$, and we are done. 


\section{ACKNOWLEDGMENTS}

I wish to thank Martin Bridson, Benson Farb, Thomas Koberda, Dan Margalit, and Ben Wieland for useful comments and conversations. I also wish to thank Dongping Zhuang for showing me how to slightly weaken the hypotheses in my original version of Theorem B.

\section{REFERENCES}

[1] J. S. Birman, Mapping class groups and their relationship to braid groups, Comm. Pure Appl. Math. 22 (1969), 213-238. MR0243519 (39:4840)

[2] M. Boggi, Fundamental groups of moduli stacks of stable curves of compact type, Geom. Topol. 13 (2009), 247-276. MR2469518

[3] M. Bridson, Semisimple actions of mapping class groups on CAT(0) spaces, LMS Lecture Notes, vol. 368, Geometry of Riemann surfaces, to appear. http://www.cambridge.org/uk/catalogue/catalogue.asp?isbn $=9780521733076$

[4] K. S. Brown, Cohomology of groups, corrected reprint of the 1982 original, Springer-Verlag, New York, 1994. MR1324339(96a:20072)

[5] R. M. Hain, Torelli groups and geometry of moduli spaces of curves, in Current topics in complex algebraic geometry (Berkeley, CA, 1992/93), 97-143, Cambridge Univ. Press, Cambridge, 1995. MR1397061 (97d:14036)

[6] N. V. Ivanov, Fifteen problems about the mapping class groups, in Problems on mapping class groups and related topics, 71-80, Proc. Sympos. Pure Math., 74, Amer. Math. Soc., Providence, RI, 2006. MR2264532(2008b:57003)

[7] D. Johnson, An abelian quotient of the mapping class group $\mathcal{I}_{g}$, Math. Ann. 249 (1980), no. 3, 225-242. MR.579103 (82a:57008)

[8] D. Johnson, The structure of the Torelli group. II. A characterization of the group generated by twists on bounding curves, Topology 24 (1985), no. 2, 113-126. MR793178 (86i:57011)

[9] D. Johnson, The structure of the Torelli group. III. The abelianization of $\mathcal{T}$, Topology 24 (1985), no. 2, 127-144. MR793179 (87a:57016)

[10] M. Korkmaz, Low-dimensional homology groups of mapping class groups: A survey, Turkish J. Math. 26 (2002), no. 1, 101-114. MR1892804 (2003f:57002)

[11] J. D. McCarthy, On the first cohomology group of cofinite subgroups in surface mapping class groups, Topology 40 (2001), no. 2, 401-418. MR.1808225 (2001m:57029)

[12] A. Putman, Cutting and pasting in the Torelli group, Geom. Topol. 11 (2007), 829-865. MR2302503 (2008c:57049)

[13] R. J. Zimmer, Ergodic theory and semisimple groups, Birkhäuser, Basel, 1984. MR776417 (86j:22014)

Department of Mathematics, Massachusetts Institute of Technology, 2-306, 77 MasSachusetts Avenue, Cambridge, Massachusetts 02139-4307

E-mail address: andyp@math.mit.edu 